\title{
Desempenhos Reprodutivo e Produtivo de Ovelhas Santa Inês Suplementadas em Diferentes Fases da Gestação ${ }^{1}$
}

\author{
Alexandre Agostinho Mexia ${ }^{2}$, Francisco de Assis Fonseca de Macedo ${ }^{3 *}$, Claudete Regina \\ Alcalde $^{3^{*}}$, Eduardo Shiguero Sakaguti ${ }^{3}$, Elias Nunes Martins ${ }^{3^{*}}$, Marilice Zundt ${ }^{2}$, Sandra Mari \\ Yamamoto4, Rosa Maria Gomes de Macedo ${ }^{3}$
}

RESUMO - Foram avaliados os comportamentos reprodutivo e produtivo de ovelhas Santa Inês, suplementadas em diferentes estádios de gestação, sendo NS = pastagem durante o dia e resíduo de fécula de mandioca no período noturno, durante a estação de monta e gestação, S46 = NS acrescido de suplementação (casca do grão de soja) do 46o dia após o início da estação de monta até a parição e S100 = NS acrescido de suplementação (casca do grão de soja) do 100o dia após o início da estação de monta até a parição. Foram utilizadas 94 ovelhas Santa Inês, acasaladas com cinco reprodutores Santa Inês e cinco Dorset. Os tratamentos não influenciaram o peso da ovelha aos 84 dias após início da suplementação (PO84), o peso da ovelha ao desmame (POD), a incidência de gestação (0 ou 1) no terço final (GEST), a taxa de parição (PAIÇÃO), taxa de natalidade (TN) e prolificidade (PROLI), sendo os valores para tais características de 49,54 kg; 38,96 kg; 0,82; 0,71; 0,88 e 1,26, respectivamente. A suplementação materna, o sexo e o grupo genético do cordeiro não influenciaram o peso ao nascimento (PCN), aos 30 dias (PC30) e aos 60 dias de idade (PC60), apresentando valores médios de 3,47; 8,26 e 12,42 kg, respectivamente. O tipo de parto influenciou o PCN e o PC30. Não houve diferença para o peso total dos cordeiros ao nascimento (PTN), peso total dos cordeiros aos 30 dias (PT30) e peso total aos 60 dias de idade (PT60) entre os tratamentos dentro de cada tipo de parto e entre os grupos genéticos. As fases de suplementação da ovelha, o grupo genético dos cordeiros e o tipo de parto não influenciaram a mortalidade, do nascimento aos 30 e 60 dias de idade. O desempenho reprodutivo de ovelhas suplementadas no período de gestação, utilizando-se $0,5 \%$ do peso vivo, com casca do grão de soja, não foi alterado.

Palavras-chave: casca do grão de soja, desenvolvimento ponderal, ovinos, prolificidade, suplementação

\section{Reproductive and Productive Development of Santa Inês Ewes Supplemented in Different Stages of Pregnancy}

\begin{abstract}
The reproductive and productive behaviour of Santa Inês ewes were evaluated as a function of supplementation in different stages of pregnancy, where NS = pasture during the day and cassava bagasse during the night over the breeding season and pregnancy, S46 = NS plus supplement (soybean hulls) from the 46th day after the beginning of the breeding season until the birth and S100 = NS plus supplement (soybean hulls) from the $100^{\text {th }}$ day after the beginning of the breeding season until the birth. Ninety-Four Santa Inês ewes were utilized, mating by five Santa Inês and five Dorset ram. The treatment did not affect ewe weight on the 84 days after the beginning of the supplementation (EW 4), weight of the ewe at the weaning (WEW), pregnancy incidence (0 or 1$)$ at the final third part of it (PREG), birth rate (BIRTH), natality rate (NR) and prolificity (PROLI), being the averages of $49.54 \mathrm{~kg} ; 0.82 ; 0.71 ; 0.88$ and 1.26 respectively. Ewes supplementation, sex and genetic group of the lamb did not influence the birth weight (LWB), by the age of 30 days (LW30) and by the 60 days (LW60). The averages were, respectively, $3.47 \mathrm{~kg} ; 8.26 \mathrm{~kg}$ and $12.42 \mathrm{~kg}$. The type of birth affected the LWB and the LW30. There was no difference for the total weight of the lambs at birth (TWB), total weight of the lambs after 30 days (TW30) and total weight of the lambs after 60 days (TW60) between the treatments, inside each type of birth and among the genetic groups. The supplementation stages of the ewe, the genetic group of the lamb and the type of birth did not influence the death rate, from the birth until the age of 30 and 60 days. The reproductive performance of the supplementeds ewes in the pregnancy period using $0.5 \%$ of live weight, with soybean hulls, was not modified.
\end{abstract}

Key Words: growth, prolificity, sheep, soybean hulls, supplementation

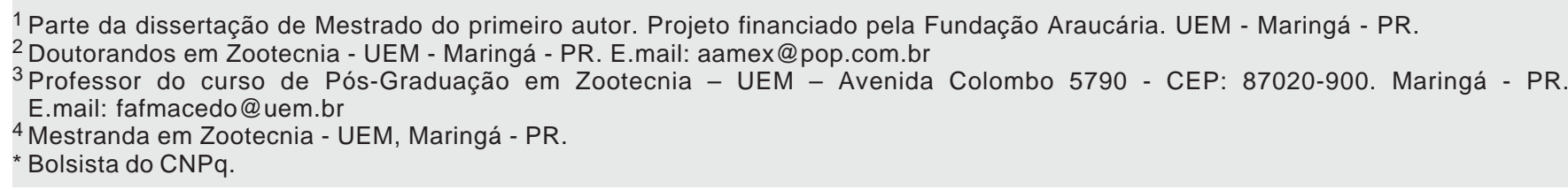




\section{Introdução}

No cenário nacional, tem aumentado o consumo de carne ovina, apresentando-se como alternativa para elevar a rentabilidade das propriedades rurais. Várias regiões do Brasil, dentre elas o Estado do Paraná, possuem condições de clima e solo que permitem a produção de carne de cordeiros, com respostas econômicas compensadoras (Macedo, 1998).

Na região sul do Brasil, os rebanhos são constituídos, em sua maioria, por raças poliéstricas estacionais, limitando o nascimento de cordeiros ao longo do ano. Raças como a Santa Inês apresentam cio durante todo ano (poliéstricas não estacionais), possibilitando três parições em dois anos, aumentando, assim, o número de cordeiros nascidos ao ano. Contudo, seus cordeiros apresentam menor velocidade de ganho de peso e carcaças de pior conformação, quando comparados às raças de corte especializadas. Tais características, segundo Dickerson (1969), poderão ser melhoradas, utilizando-se reprodutores das raças de corte, pois as diferenças genéticas existentes entre as raças podem ser utilizadas, no sentido de adequar tipo de animal e ambiente, para aumentar a eficiência dos sistemas de produção.

O desempenho reprodutivo de um rebanho e a taxa de crescimento dos animais estão entre os principais componentes responsáveis pelo sucesso da produção. Somente o aumento do número de cordeiros nascidos não é suficiente para o incremento da ovinocultura de corte. O nascimento de animais com maior velocidade de ganho de peso é necessário, o que pode ser obtido com cruzamento e manejo nutricional adequados às ovelhas em gestação.

A gestação da ovelha tem duração de aproximadamente 150 dias, sendo que nos 40-50 dias finais de gestação ocorrem cerca de $70 \%$ do crescimento do feto, momento de ingressar com estratégias de manejo que garantam correto aporte de nutrientes às ovelhas. $\mathrm{O}$ aumento das exigências nesta fase pode, de acordo com Susin (1996), ser atendido com concentrados, sabendose que a maioria dos volumosos é de baixa qualidade.

A nutrição inadequada da ovelha durante a gestação poderá limitar a capacidade de crescimento pós-natal dos músculos esqueléticos dos cordeiros (Greenwood et al., 2000). Uma ração pobre em energia reduz a fertilidade, diminui o ganho de peso e a produção de leite, mas o fornecimento excessivo de energia, além de conduzir a acúmulos de gordura, pode prejudicar a eficiência de produção. A proteína, por sua vez, é o principal constituinte corporal do animal, sendo vital para os processos de manutenção, crescimento e reprodução (Estrada, 2000).

Sabe-se que inúmeras pesquisas sobre o comportamento reprodutivo da raça Santa Inês estão sendo realizadas nas regiões sudeste e nordeste do país. No entanto, no Paraná, ainda são escassos os trabalhos com esta raça, os quais são fundamentais para que o criador possa fazer sua opção de maneira acertada.

O objetivo deste experimento foi avaliar os comportamentos reprodutivo e produtivo de ovelhas Santa Inês, suplementadas em diferentes estádios de gestação e o desenvolvimento ponderal de seus cordeiros, puros e cruzados com a raça Dorper.

\section{Material e Métodos}

O experimento foi realizado no Centro de Pesquisa do Arenito, da Universidade de Maringá, no município de Cidade Gaúcha, Noroeste do Paraná. Esta região situa-se a $23^{\circ} 25^{\prime}$ de latitude Sul, $51^{\circ} 55^{\prime}$ ' de longitude Oeste e 554,9 m de altitude. O clima predominante, segundo Corrêa (1996), é classificado como subtropical úmido mesotérmico com verões quentes, geadas pouco freqüentes, com tendências de concentração de chuvas no verão.

Foram utilizadas 94 ovelhas Santa Inês, com peso médio de 44,31 kg, adquiridas entre outubro de 2000 a janeiro de 2001, de propriedades particulares, num total de quatro lotes. Os animais permaneceram em adaptação no local, até o início da estação de monta. Quarenta e sete ovelhas foram cobertas com cinco reprodutores Santa Inês e as outras 47 , com cinco carneiros Dorset. A estação de monta teve início em maio de 2001, com 45 dias de duração. Os nascimentos ocorreram de outubro a novembro do mesmo ano.

Como raça paterna, zootecnicamente poderia ser utilizada a raça Dorper ou Dorset, porque ciclam o ano inteiro e possuem boa conformação de carcaça. Entretanto, pela baixa disponibilidade de reprodutores da raça Dorper, utilizaram-se carneiros da raça Dorset.

Foram estimadas a disponibilidade de massa de forragem, a determinação das proporções da planta (folhas, colmos e material morto) e a composição química da folha do capim-Tanzânia (Panicum maximum Jacq.), através de corte das plantas rente ao solo. Encontrou-se disponibilidade de $1531 \mathrm{~kg} \mathrm{MS} / \mathrm{ha}$, em média, no período entre a estação de monta e o final dos nascimentos, sendo $64 \%$ de folhas, $28 \%$ de colmos e $8 \%$ de matéria morta.

R. Bras. Zootec., v.33, n.3, p.658-667, 2004 
Durante o experimento, o rebanho permaneceu na mesma pastagem no período diurno, sendo recolhido em instalações cobertas, com piso ripado e suspenso, no período noturno, para proteção contra predadores e menor exposição à infecção por helmintos parasitas.

Foram utilizados três tratamentos, sendo um controle, em que os animais somente receberam pasto e resíduo de fécula de mandioca; para os demais tratamentos, houve suplementação com casca do grão de soja, diferindo por fase de gestação.

O resíduo de fécula de mandioca foi distribuído, a $0,8 \%$ do peso vivo na matéria seca, a todas as ovelhas após serem recolhidas às instalações, e a casca do grão de soja fornecida na base de $0,5 \%$ do peso vivo, somente no período da manhã, antes dos animais serem soltos ao campo. As ovelhas tiveram acesso à mistura mineral, em outros cochos.

Os tratamentos consistiram de:

NS = pastagem durante o dia e resíduo de fécula de mandioca no período noturno.

S46 = pastagem durante o dia e resíduo de fécula de mandioca no período noturno acrescido de suplementação (casca do grão de soja) do 46으 dia após o início da estação de monta até a parição.

S100 = pastagem durante o dia e resíduo de fécula de mandioca no período noturno acrescido de suplementação (casca do grão de soja) do $100^{\circ}$ dia após o início da estação de monta até a parição.

As amostras de alimentos e da ração do creep feeding foram analisadas no Laboratório de Nutrição Animal (Departamento de Zootecnia-UEM), segundo metodologias descritas por Silva (1990). Os valores com base na matéria seca de proteína bruta (PB), fibra bruta (FB), extrato etéreo (EE), cinzas e energia bruta (EB) da casca do grão de soja, da folha de Tanzânia e do resíduo de fécula de mandioca, encontram-se na Tabela 1.

A infecção por endoparasitas foi acompanhada por contagem de ovos por grama de fezes (OPG), sendo as ovelhas tratadas com anti-helmíntico, quando essa foi superior a 500 .

\section{Manejo dos cordeiros pós-parição}

Os cordeiros tiveram acesso, em creep feeding, a uma dieta formulada para ganho de peso médio de 230 gramas/dia (NRC, 1985), cuja composição química e percentual podem ser vizualizadas na Tabela 2 .

Na primeira semana, as ovelhas permaneceram confinadas com seus cordeiros durante as 24 horas do dia. Posteriormente, até a terceira semana, somente as ovelhas tiveram acesso à pastagem 4 horas/dia, no período vespertino. Da quarta semana até o desmame (aos 60 dias), as ovelhas permaneceram durante o dia em pastagem, sendo recolhidas no final da tarde.

Os cordeiros foram vacinados contra carbúnculo, gangrena, enterotoxemia e ectima contagioso.

As ovelhas foram pesadas no início e término da estação de monta, posteriormente a cada 28 dias até a parição e no desmame. Os cordeiros foram numerados e pesados ao nascimento, aos 30 dias e aos 60 dias de idade (desmame).

Foi avaliado o efeito da suplementação nas diferentes fases de gestação, grupo genético dos cordeiros, sexo, tipo de parto, peso da ovelha e escore de condição corporal da ovelha.

As características observadas foram: peso do cordeiro ao nascimento (PCN), aos 30 dias de idade (PC30) e aos 60 dias (PC60), peso total dos cordeiros ao nascimento (PTN), de acordo com a fase de suplementação materna, tipo de parto (simples ou duplo) e grupo genético (Santa Inês ou 1/2 Dorset + 1/2

Tabela 1 - Composição química e bromatológica dos alimentos, base na matéria seca ${ }^{1}$ Table 1 - Chemical composition of feedstuffs, in dry matter basis ${ }^{1}$

\begin{tabular}{|c|c|c|c|c|c|}
\hline $\begin{array}{l}\text { Alimentos } \\
\text { Feedstuffs }\end{array}$ & $\begin{array}{c}\mathrm{PB}(\%) \\
C P\end{array}$ & $\begin{array}{c}\mathrm{EE}(\%) \\
E E\end{array}$ & $\begin{array}{l}\mathrm{FDN}(\%) \\
\quad N D F\end{array}$ & $\begin{array}{l}\text { Cinzas (\%) } \\
\text { Ash }\end{array}$ & $\begin{array}{c}\mathrm{EB}(\mathrm{Mcal} / \mathrm{kg}) \\
G E\end{array}$ \\
\hline $\begin{array}{l}\text { Casca do grão de soja } \\
\text { Soybean hulls }\end{array}$ & 10,73 & 1,89 & 59,27 & 3,68 & 4,06 \\
\hline $\begin{array}{l}\text { Folha de Tanzânia } \\
\text { Tanzania leaf }\end{array}$ & 7,12 & 2,31 & 68,74 & 8,41 & 4,10 \\
\hline $\begin{array}{l}\text { Resíduo de fécula de mandioca } \\
\text { Cassava bagasse }\end{array}$ & 1,87 & 0,55 & 9,34 & 1,68 & 3,78 \\
\hline
\end{tabular}

${ }^{1} \mathrm{~PB}=$ proteína bruta, $\mathrm{EE}$ = extrato etéreo, FDN = fibra detergente neutro e EB = energia bruta.

${ }^{1} C P=$ crude protein, $E E=$ ether extract, $N D F=$ neutral detergent fiber and $G E=$ gross energy. 
Santa Inês), peso total dos cordeiros aos 30 dias (PT30) e peso total aos 60 dias de idade (PT60), peso da ovelha aos 84 dias após início da suplementação (PO84), peso da ovelha ao desmame (POD), incidência de gestação (0 para não gestante e 1 para gestante) no terço final (GEST) e taxa de parição (0 para as que não pariram e 1 para as que pariram) (PARIÇÃO), taxa de natalidade do rebanho total (TN), número de cordeiros ao nascimento por ovelha parida (PROLI), número de cordeiros por ovelha com cria viva aos 30 dias (NC30) e número de cordeiros por ovelha com cria viva aos 60 dias (NC60), número de cordeiros mortos por ovelha do nascimento aos 30 dias (MN30) e do nascimento aos 60 dias (MN60).

Os dados foram analisados pelo programa computacional SAS (1992), seguindo o procedimento GLM para PCN, PC30, PC60, PTN, PT30, PT60, PO84, POD.

Utilizou-se o procedimento GENMOD para GEST, PARIÇÃO e TN, assumindo distribuição binomial e função de ligação logística, entretanto, para PROLI, NC30, NC60, MN30 e MN60, assumiu-se distribuição de Poisson e função de ligação log.

O modelo utilizado nas análises para as características PCN, PC30 e PC60 foi:

$$
\begin{aligned}
& Y_{i j k l m}=\mu+T_{i}+G G_{j}+T G G_{i j}+S_{k}+T P_{l}+ \\
& b 1\left(P O I_{i j k l m}-M P O I\right)+e_{i j k l m}
\end{aligned}
$$

em que $Y_{i j k l m}=$ valor observado da variável estudada no indivíduo $m$, nascido de parto $l$, do sexo $k$, pertencente ao grupo genético $j$, cuja ovelha recebeu na gestação a suplementação $i ; \mu=$ constante geral; $T_{i}=$ efeito do tipo de suplementação $i$ na gestação ; sendo $i=1,2$ e 3; $G G_{j}=$ efeito do grupo genético $j$ do cordeiro; sendo $j=1$ e 2; $T G G_{i j}=$ efeito da interação entre o tratamento $i$ e o grupo genético $j ; S_{k}=$ efeito do sexo $k$; sendo $k=1$ e $2 ; T P_{l}=$ efeito do tipo de parto $l$, sendo $l=1$ e 2 ; $b 1$ = coeficiente de regressão linear para a característica em função do peso inicial da ovelha; $P O I_{i j k l m}=$ peso inicial da ovelha, mãe do animal $m$, nascido de parto $l$, do sexo $k$, pertencente ao grupo genético $j$, cuja ovelha recebeu na gestação a suplementação $i ; M P O I=$ média dos pesos das ovelhas; $\mathrm{e}_{i j k l m}=$ erro aleatório associado a cada observação $Y_{i j k l m}$.

Para as características PTN, PT30 e PT60, foi utilizado o seguinte modelo:

$$
\begin{gathered}
Y_{i j k l}=\mu+T_{i}+G G_{j}+T G G_{i j}+T P_{k}+T T P_{i k}+b 1 \\
\left(P O I_{i j k l}-P O I\right)+b 2\left(E I_{i j k l}-M E\right)+e_{i j k l}
\end{gathered}
$$

em que $Y_{i j k l m}=$ valor observado da variável estudada no grupo de indivíduos $l$, nascido de parto $k$, pertencente ao grupo genético $j$, cuja ovelha recebeu na gestação a suplementação $i$; $T T P_{i k}=$ efeito da interação entre o tratamento e tipo de parto; $b 2$ = coeficiente de regressão linear para a característica em função do escore corporal inicial da ovelha; $E I_{i j k l}=$ escore corporal inicial da ovelha, mãe do grupo de indivíduos $l$, tendo nascido de parto $k$, pertencente ao grupo genético $j$, cuja ovelha recebeu na gestação a suplementação $i ; M E=$ média dos escores corporais das ovelhas; $\mathrm{e}_{i j k l}=$ erro aleatório associado a cada observação $Y_{i j k l}$

Os demais efeitos já foram descritos no modelo anterior.

Para a característica PO84 foi utilizado o seguinte modelo:

$$
\begin{gathered}
Y_{i j}=\mu+T_{i}+b 1\left(P O I_{i j}-P O I\right)+b 2\left(E I_{i j}-M E\right)+ \\
b 3\left(D G_{i j}+M D G\right)+e_{i j}
\end{gathered}
$$

Tabela 2 - Composição percentual e química da ração dos cordeiros

Table 2 - Percentual and chemical composition of lambs diet

\begin{tabular}{lc}
\hline $\begin{array}{l}\text { Ingredientes } \\
\text { Ingredients }\end{array}$ & $\% \mathrm{MS}$ \\
\hline $\begin{array}{l}\text { Milho } \\
\text { Corn }\end{array}$ & 54,86 \\
$\begin{array}{l}\text { Farelo de soja } \\
\text { Soybean meal }\end{array}$ & 23,93 \\
$\begin{array}{l}\text { Farelo de trigo } \\
\text { Wheat meal }\end{array}$ & \\
$\begin{array}{l}\text { Feno de aveia } \\
\text { Oat hay } \\
\text { Mistura mineral } \\
\begin{array}{l}\text { Mineral mixture } \\
\text { Lisina }\end{array}\end{array}$ \\
$\begin{array}{l}\text { Lysine } \\
\text { Metionina } \\
\text { Methionine }\end{array}$ \\
\hline
\end{tabular}

Nutrientes

Nutrients

Matéria seca (\%)

91,20

Dry matter (\%)

Proteína bruta (\%)

18,20

Crude protein (\%)

Cálcio (\%)

1,46

Calcium (\%)

Fósforo (\%)

Phosporous (\%)

R. Bras. Zootec., v.33, n.3, p.658-667, 2004 
em que $Y_{i j}=$ valor observado da variável estudada na ovelha $j$ que recebeu na gestação a suplementação $i$; $b 3$ = coeficiente de regressão linear para a característica em função do dia de gestação; $D G_{i j}=$ dia de gestação inicial da ovelha $j$, que recebeu na gestação a suplementação $i ; M D G$ = média dos dias de gestação; $e_{i j}=$ erro aleatório associado a cada observação $\mathrm{Y}_{i j}$.

Os demais efeitos já foram descritos nos modelos anteriores.

Para as características POD, GEST, NASC e TN, foi utilizado o seguinte modelo:

$$
Y_{i j}=\mu+T_{i}+b 1\left(P_{i j}-P O I\right)+b 2\left(E I_{i j}-M E\right)+e_{i j}
$$

em que $Y_{i j}=$ valor observado da variável estudada na ovelha $j$, que recebeu na gestação a suplementação $i$; $e_{i j}=$ erro aleatório associado a cada observação $Y_{i j}$.

Os demais efeitos já foram descritos nos modelos anteriores.

Para as características PROLI, NC30, NC60, MN-30 e MN-60, foi utilizado o seguinte modelo:

$$
\begin{gathered}
Y_{i j}=\mu+T_{i}+G G_{j}+b 1\left(P O I_{i j k}-P O I\right)+b 2\left(E I_{i j k}\right. \\
-M E)+e_{i j k}
\end{gathered}
$$

em que $Y_{i j k l m}=$ valor observado da variável estudada no indivíduo $k$, pertencente ao grupo genético $j$, cuja ovelha recebeu na gestação a suplementação $i$; $e_{i j k}=$ erro aleatório associado a cada observação $Y_{i j k \text {. }}$

Os demais efeitos já foram descritos nos modelos anteriores.

\section{Resultados e Discussão}

A suplementação nas diferentes fases de gestação (Tabela 3) não influenciou ( $\mathrm{P} \geq 0,05)$ o peso da ovelha aos 84 dias após início da suplementação (PO84), peso da ovelha ao desmame (POD), incidência de gestação no terço final (GEST), taxa de parição (PARIÇÃO), taxa de natalidade (TN), prolificidade (PROLI), o número de cordeiros por ovelha com cria viva aos 30 dias (NC30) e número de cordeiros por ovelha com cria viva aos 60 dias (NC60).

Houve pequeno aumento no valor absoluto do PO84, POD e menor porcentagem de aborto em relação às ovelhas do tratamento S46, provavelmente pela suplementação das ovelhas durante a gestação (Tabela 3).

O peso da ovelha exerceu influência linear crescente sobre o POD e PO84, sendo esta última característica influenciada, também, pelo dia de gestação.

Os valores médios encontrados para as variáveis

Tabela 3 - Valores médios estimados para peso da ovelha aos 84 dias, após início da suplementação (PO84), peso da ovelha ao desmame (POD), incidência de gestação (0 ou 1) no terço final (GEST), taxa de parição (PARIÇÃO), taxa de natalidade (TN), prolificidade (PROLI), número de cordeiros por ovelha com cria viva aos 30 dias (NC30) e número de cordeiros por ovelha com cria viva aos 60 dias (NC60), de acordo com o tipo de suplementação materna

Table 3 - Estimated mean values to ewe weight on the 84 days after the beginning of the supplementation (EW84), weight of the ewe at the weaning (WEW), pregnancy incidence (0 or 1) at the final third part (PREG), birth rate (BIRTH), natality rate (NR), prolificity (PROLI), number of lamb per ewe with alive offspring after 30 days (NL30) and number of lamb per ewe with alive offspring after 60 days (NL60), according to the type of mother supplementation

\begin{tabular}{lcccccccc}
\hline $\begin{array}{l}\text { Tratamento } \\
\text { Treatment }\end{array}$ & PO84 & POD & GEST & PARIÇÃO & TN & PROLI & NC30 & NC60 \\
\hline NS $^{1}(28)$ & 47,88 & 37,45 & 0,86 & 0,68 & 0,84 & 1,32 & 1,14 & 1,08 \\
S46 $^{1}(33)$ & 50,04 & 39,26 & 0,80 & 0,77 & 0,88 & 1,19 & 1,16 & 1,20 \\
S100 $^{1}$ (32) & 48,66 & 37,88 & 0,81 & 0,69 & 0,90 & 1,26 & 1,07 & 1,07 \\
Média geral & 49,54 & 38,96 & 0,82 & 0,71 & 0,88 & 1,26 & 1,13 & 1,12 \\
General average & & & & & & & & \\
CV ou DEV & & & & & & & & \\
C,02 & 10,12 & 86,95 & 108,63 & 64,28 & 9,45 & 4,13 & 3,41 \\
\hline
\end{tabular}

${ }^{1} \mathrm{NS}=$ pastagem durante o dia e resíduo de fécula de mandioca no período noturno, S46= NS + suplementação (casca do grão de soja) do 46ํ dia após o início da estação de monta até a parição e S100= NS + suplementação (casca do grão de soja) do 100 o dia após o início da estação de monta até a parição.

* CV = coeficiente de variação para P084 e POD; DEV = deviancia para GEST, PARIÇÃO, TN, PROLI, NC30 e NC60.

${ }^{1} \mathrm{NS}=$ pasture during the day and cassava bagasse during the night, S46= NS + supplement (soybean hulls) from the $46^{\text {th }}$ day after the beginning of the breeding season until the birth and S100 $=N S+$ supplement (soybean hulls) from the $100^{\text {th }}$ day after the beginning of the breeding season until the birth.

* CV = coefficient variation to EW84 and WEW; DEV = deviance to PREG, BIRTH, NR, PROLI, LC30 and NL60.

R. Bras. Zootec., v.33, n.3, p.658-667, 2004 
analisadas em relação ao desempenho reprodutivo das ovelhas Santa Inês na região noroeste do Paraná foram de $25 \%$ de partos duplos e $75 \%$ de parto simples, relação macho:fêmea ao nascimento 61:39.

No nordeste brasileiro, Pereira et al. (1998) observaram, na raça Santa Inês, valores de 90,32\% para taxa de parição, sendo 58,4\% de partos simples e $41,6 \%$ de partos múltiplos, com prolificidade de 1,48 , indicando uma média de três partos a cada dois anos. Vinagre et al. (1992) obtiveram valor semelhante para taxa de parição (88\%), porém, para índice de prolificidade, porcentagem de partos simples e partos múltiplos, os valores encontrados foram 1,3; $77,3 \%$ e $22,7 \%$, respectivamente. Machado et al. (1999) observaram para taxa de parição de 98,5\%, valor mais alto que os demais citados.

A menor eficiência reprodutiva encontrada neste experimento, em relação aos da literatura, pode ser justificada pela heterogeneidade do plantel materno, adquirido de várias propriedades no Estado, meses antes do início da estação de monta e pelo curto intervalo de observações.

O escore corporal no início do experimento tomado como co-variável não afetou $(\mathrm{P} \geq 0,05)$ os índices reprodutivos avaliados. Lobato \& Barcellos (1988), avaliando o desempenho reprodutivo de novilhas, inferiram que, apesar de não significativa, a condição corporal no início do acasalamento favoreceu o desempenho reprodutivo.

O peso dos cordeiros ao nascimento (PCN), aos 30 dias de idade (PC30) e ao desmame (PC60), foram semelhantes $(P \geq 0,05)$ entre os períodos de suplementação das ovelhas (Tabela 4). O peso ao nascer merece maior atenção em relação aos parâmetros reprodutivos, pois cordeiros nascidos pequenos e débeis, normalmente, têm menores possibilidades de sobrevivência, devido à dificuldade de procurar alimento.

Silveira et al. (1992), trabalhando com ovelhas Ideal, suplementadas com diferentes dietas durante toda a gestação, também não encontraram diferença para PCN. Entretanto, Rodrigues et al. (1989) verificaram diferenças no peso dos cordeiros ao nascimento, trabalhando com ovelhas alimentadas, de acordo com as exigências do NRC, com azevém e com campo nativo. Salomão et al. (1996), suplementando ovelhas com mistura protéico-energética-mineral no terço final de gestação, observaram que o lote suplementado produziu cordeiros significativamente maiores ao nascer, sendo que os cordeiros machos nascidos de parto simples, com e sem suplementação materna, pesaram 4,4 e 3,6 kg, respectivamente, e as fêmeas nascidas de parto simples, com e sem suplementação materna, pesaram 4,4 e 3,7 kg, respectivamente.

Os valores para PCN e PC30 foram semelhantes aos encontrados por Barbiere et al. (1991), Lima et al. (1991) e Silva et al. (1995), trabalhando com ovelhas Santa Inês. Em relação ao PC60, os valores citados por Santana et al. (2001) e os demais autores citados anteriormente ficaram, porém, um pouco abaixo dos obtidos no presente trabalho.

$\mathrm{O}$ peso dos cordeiros ao nascimento, aos 30 e 60 dias de idade não foi influenciado $(\mathrm{P} \geq 0,05)$ pelo sexo, concordando com os resultados encontrados por Lima et al. (1987), trabalhando com PCN e peso aos 56 dias de idade; por Salomão et al. (1996), avaliando PCN; e por Machado et al. (1999), estudando peso do nascimento ao desmame. Lôbo et al. (1992), Oliveira (1992) e Silva et al. (1995) evidenciaram, entretanto, a superioridade de peso dos machos em relação às fêmeas, para as raças Morada Nova e Santa Inês.

Não houve efeito $(P \geq 0,05)$ do grupo genético dos cordeiros em relação ao peso vivo até a desmama. Estes resultados estão de acordo com os achados de Lima et al. (1991) e de Souza Júnior et al. (2000). Machado et al. (1999), porém, não encontraram efeito apenas para peso à desmama entre diferentes grupos genéticos. No período de aleitamento, os cordeiros não expressam as diferenças genotípicas esperadas, pois a curva de crescimento nesta fase acompanha a curva de lactação da matriz, que está mais intimamente ligada à oferta nutricional durante a lactação que ao genótipo da cria (Figueiró, 1989).

Wolf et al. (1980) encontraram efeito de genótipo para pesos ao nascimento, avaliando os genótipos Dorset Down, Oxford Down, Suffolk, Ile de France, Oldemburg e Texel. O mesmo resultado foi obtido por Butter-Hogg (1984), que estudou Southdown e Cluns, e por Cameron \& Smith (1984), que pesquisaram Border Leicester, Blueface Leicester e Abro Damline.

$\mathrm{O}$ tipo de parto influenciou $(\mathrm{P}<0,05)$ o peso dos cordeiros ao nascer e aos 30 dias de idade (Tabela 4). Este último peso pode ser explicado pelo fato de o consumo de leite ser um importante fator para o crescimento do cordeiro nas primeiras semanas de vida. Church (1984) e Ramsey et al. (1994) alegam que, embora ovelhas que parem gêmeos apresentem maior produção de leite, não chega a ser o dobro da produção de uma ovelha de parto simples. Assim, os cordeiros consumirão menor quantidade de leite, mas

\section{R. Bras. Zootec., v.33, n.3, p.658-667, 2004}


a superioridade do ganho de peso de cordeiros de parto simples diminuirá no final da lactação, provavelmente devido ao maior consumo de ração pelos cordeiros de parto gemelar (Peeters et al., 1996).

Vários autores, estudando as raças deslanadas, constataram a influência do tipo de parto sobre o peso ao nascimento e demais idades, destacando-se os trabalhos de Oliveira (1992), de Muniz et al. (1997) e de Silva et al. (1998).

Observa-se na Tabela 5 que não houve diferença $(\mathrm{P} \geq 0,05)$ para PTN, PT30 e PT60 entre os tratamentos dentro de cada tipo de parto (simples ou duplo) e grupo genético. Apenas o PT30 dias de idade sofreu influência $(\mathrm{P} \leq 0,05)$ do peso inicial da ovelha, com médias de pesos totais estimados maiores em ovelhas com parto duplo.

Machado et al. (1999) também não encontraram diferença para o PTN em relação ao grupo genético, contudo, devido à maior mortalidade nos cordeiros F1 Hampshire Down, estes apresentaram menor peso total ao desmame, quando comparado aos grupos genéticos Santa Inês, Ile de France, Suffolk e Texel.

As fases de suplementação da ovelha, o grupo

Tabela 4 - Valores médios estimados para peso do cordeiro ao nascimento (PCN), aos 30 dias de idade (PC30) e aos 60 dias (PC60), de acordo com o tipo de suplementação materna, grupo genético (Santa Inês ou $1 / 2$ Dorset $1 / 2$ Santa Inês) e tipo de parto (simples ou duplo)

Table 4 - Estimated mean values to weight of the lambs at birth weight (LWB), at 30 days ( $L W 30$ ) and at 60 days ( $L W 60$ ) according to the type of mother supplementation, genetic group (Santa Inês or $1 / 2$ Dorset $+1 / 2$ Santa Inês) and the type of birth (simple or double)

\begin{tabular}{|c|c|c|c|c|}
\hline $\begin{array}{l}\text { Efeito } \\
\text { Effect }\end{array}$ & & $\begin{array}{l}\text { PCN } \\
L W B\end{array}$ & $\begin{array}{l}\text { РC30 } \\
L W 30\end{array}$ & $\begin{array}{l}\text { PC60 } \\
L W 60\end{array}$ \\
\hline \multicolumn{5}{|l|}{ Tratamento } \\
\hline \multicolumn{5}{|l|}{ Treatment } \\
\hline $\mathrm{NS}^{1}$ & & 3,32 & 8,12 & 12,24 \\
\hline $\mathrm{S} 46^{1}$ & & 3,60 & 7,93 & 12,22 \\
\hline $\mathrm{S} 100^{1}$ & & 3,32 & 8,23 & 12,83 \\
\hline \multicolumn{5}{|c|}{ Grupo genético/Sexo } \\
\hline \multicolumn{5}{|c|}{ Genetic group/Sex } \\
\hline & Fêmea & 3,49 & 8,03 & 12,52 \\
\hline \multirow[t]{2}{*}{ Dorset } & Female & & & \\
\hline & $\begin{array}{c}\text { Macho } \\
\text { Male }\end{array}$ & 3,45 & 8,05 & 12,91 \\
\hline \multirow[t]{3}{*}{ Santa Inês } & Fêmea & 3,29 & 8,43 & 12,75 \\
\hline & Female & & & \\
\hline & $\begin{array}{c}\text { Macho } \\
\text { Male }\end{array}$ & 3,43 & 7,85 & 11,54 \\
\hline \\
\hline \multicolumn{5}{|l|}{ Type of birth } \\
\hline Simples & & $3,71 a$ & $9,19 a$ & 13,02 \\
\hline Simple & & & & \\
\hline Duplo & & $3,12 b$ & $6,99 b$ & 11,84 \\
\hline Double & & & & \\
\hline Média Geral & & 3,47 & 8,26 & 12,42 \\
\hline \multicolumn{5}{|l|}{ General average } \\
\hline $\mathrm{CV} *$ & & 17,55 & 21,14 & 25,56 \\
\hline
\end{tabular}

${ }^{1} \mathrm{NS}=$ pastagem durante o dia e resíduo de fécula de mandioca no período noturno, S46= NS + suplementação (casca do grão de soja) do $46^{\circ}$ dia após o início da estação de monta até a parição e S100= NS + suplementação (casca do grão de soja) do $100^{\circ}$ dia após o início da estação de monta até a parição.

${ }^{*} \mathrm{CV}=$ coeficiente de variação para PCN, PC30 e PC60.

${ }^{1} \mathrm{NS}=$ pasture during the day and cassava bagasse during the night, $S 46=N S+$ supplement (soybean hulls) from the 46th day after the beginning of the breeding season until the birth and $\mathrm{S} 100=N S+$ supplement (soybean hulls) from the 100th day after the beginning of the breeding season until the birth.

${ }^{*} \mathrm{CV}=$ Variation coefficient to $L W B, L W 30$ and $L W 60$.

R. Bras. Zootec., v.33, n.3, p.658-667, 2004 
genético dos cordeiros e o tipo de parto não influenciaram $(P \geq 0,05)$ a mortalidade do nascimento aos 30 dias de idade e do nascimento aos 60 dias (Tabela 6). A apresentação destes valores são de grande importância prática, apesar de serem iguais estatisticamente.

Os valores de mortalidade obtidos neste trabalho foram maiores aos encontrados por Silva \& Arruda (1988), que, avaliando ovinos Santa Inês, mantidos em pastagem raleada e não raleada no semi-árido
Paraibano, observaram mortalidade de 22,66 e 22,08\%, respectivamente. Maiores também que aquele observado por Mcmanus (1995), que encontrou valor médio de $28 \%$, para mortalidade até desmama, quando avaliou rebanho Santa Inês, na região de Brasília. Contudo, os achados para mortalidade até o desmame equivalem aos relatados por Vinagre et al. (1989), que verificaram mortalidade de $39,73 \%$ em cordeiros Santa Inês, do nascimento ao desmame, nas condições do Agreste Paraibano.

Tabela 5 - Valores médios estimados de peso total dos cordeiros ao nascimento (PTN), peso total dos cordeiros aos 30 dias (PT30) e peso total aos 60 dias de idade (PT60), de acordo com o tipo de suplementação materna, tipo de parto (simples ou duplo) e grupo genético (Santa Inês ou 1/2 Dorset $1 / 2$ Santa Inês)

Table 5 - Estimated mean values of total weight of the lambs at birth (TWB), total weight of the lambs at 30 days (TW30) and total weight at 60 days (TW60), according to the type of mother supplementation, the type of birth (simple or double) and genetic group (Santa Inês or $1 / 2$ Dorset $1 / 2$ Santa Inês)

\begin{tabular}{|c|c|c|c|c|}
\hline \multicolumn{2}{|l|}{$\begin{array}{l}\text { Efeito } \\
\text { Effect }\end{array}$} & $\begin{array}{l}\text { PTN } \\
\text { TWB }\end{array}$ & $\begin{array}{l}\text { PT30 } \\
\text { TW30 }\end{array}$ & $\begin{array}{l}\text { PT60 } \\
\text { TW60 }\end{array}$ \\
\hline \multicolumn{5}{|c|}{ Tipo de parto/Tratamento } \\
\hline \multicolumn{5}{|c|}{ Type of birth/Treatment } \\
\hline $\begin{array}{l}\text { Simples } \\
\text { Simple }\end{array}$ & $\mathrm{NS}^{1}$ & 3,58 & 7,66 & 12,04 \\
\hline & $\mathrm{S} 46^{1}$ & 3,85 & 8,29 & 11,83 \\
\hline & $\mathrm{S} 100^{1}$ & 3,60 & 7,70 & 12,08 \\
\hline \multicolumn{2}{|c|}{$\begin{array}{l}\text { Média do Parto Simples } \\
\text { Average of Simple Birth }\end{array}$} & 3,68 & 7,89 & 11,98 \\
\hline \multicolumn{5}{|l|}{ Duplo } \\
\hline \multirow[t]{3}{*}{ Double } & $\mathrm{NS}^{1}$ & 6,11 & 12,78 & 17,57 \\
\hline & $\mathrm{S} 46^{1}$ & 6,58 & 12,43 & 23,06 \\
\hline & $\mathrm{S} 100^{1}$ & 6,01 & 14,66 & 26,39 \\
\hline \multirow{2}{*}{\multicolumn{2}{|c|}{$\begin{array}{l}\text { Média do Parto Duplo } \\
\text { Average of Double Birth }\end{array}$}} & 6,24 & 13,29 & 22,34 \\
\hline & & & & \\
\hline \multicolumn{5}{|c|}{ Grupo Genético } \\
\hline \multicolumn{5}{|l|}{ Genetic Group } \\
\hline \multicolumn{2}{|l|}{ Dorset } & 5,01 & 10,93 & 18,11 \\
\hline \multicolumn{2}{|l|}{ Santa Inês } & 4,91 & 10,25 & 16,21 \\
\hline \multirow{2}{*}{\multicolumn{2}{|c|}{$\begin{array}{c}\text { Média Geral } \\
\text { General Average }\end{array}$}} & 4,96 & 10,59 & 17,16 \\
\hline & & & & \\
\hline \multicolumn{2}{|c|}{$\mathrm{CV}^{*}$} & 15,47 & 20,01 & 28,07 \\
\hline
\end{tabular}

${ }^{1} \mathrm{NS}=$ pastagem durante o dia e resíduo de fécula de mandioca no período noturno, $\mathrm{S} 46=\mathrm{NS}+$ suplementação (casca do grão de soja) do $46^{\circ}$ dia após o início da estação de monta até a parição e S100= NS + suplementação (casca do grão de soja) do $100^{\circ}$ dia após o início da estação de monta até a parição.

* CV = coeficiente de variação para PTN, PT30 e PT60.

${ }^{1} N S=$ pasture during the day and cassava bagasse during the night, $S 46=N S+$ supplement (soybean hulls) from the 46th day after the beginning of the breeding season until the birth and $\mathrm{S} 100=N S+$ supplement (soybean hulls) from the 100th day after the beginning of the breeding season until the birth.

${ }^{*} C V=$ Variation coefficient to TWB, TW30 and TW60.

R. Bras. Zootec., v.33, n.3, p.658-667, 2004 
Tabela 6 - Valores médios estimados de porcentagem de cordeiros mortos por ovelha do nascimento aos 30 dias (MN30) e do nascimento aos 60 dias (MN60), de acordo com o tipo de suplementação materna, grupo genético (Santa Inês ou $1 / 2$ Dorset $1 / 2$ Santa Inês) e tipo de parto (simples ou duplo)

Table 6 - Estimated mean values of percentage of the lambs death per ewes from the birth at 30 days (DB30) and from the birth until 60 days (DB60), according to the type of mother supplementation, genetic group (Santa Inês or $1 / 2$ Dorset $1 / 2$ Santa Inês) and type of birth (simple or double)

\begin{tabular}{rcc}
\hline Efeito & MN30(\%) & MN60(\%) \\
Effect & DB30 (\%) & DB60 (\%) \\
\hline
\end{tabular}

Tratamento

Treatment

$\begin{array}{lll}\mathrm{NS}^{1} & 34,26 & 53,89 \\ \mathrm{~S}^{1} 6^{1} & 27,09 & 37,18 \\ \mathrm{~S}^{100} & 23,78 & 23,93\end{array}$

Grupo genético

Genetic Group

$\begin{array}{ccc}\text { Dorset } & 18,77 & 25,77 \\ \text { Santa Inês } & 41,92 & 51,21\end{array}$

Tipo de parto

Type of birth

\begin{tabular}{ccc}
$\begin{array}{c}\text { Simples } \\
\text { Simple } \\
\text { Duplo } \\
\text { Double }\end{array}$ & 20,76 & 27,39 \\
\hline Média geral & 41,14 & 48,68 \\
General average & 30,15 & 39,02 \\
DEV $^{*}$ & 40,57 & 41,63 \\
\hline
\end{tabular}

${ }^{1} \mathrm{NS}$ = pastagem durante o dia e resíduo de fécula de mandioca no período noturno, S46= NS + suplementação (casca do grão de soja) do 46으 dia após o início da estação de monta até a parição e S100= NS + suplementação (casca do grão de soja) do 100 o dia após o início da estação de monta até a parição. * DEV = Deviância para MN30, MN60.

${ }^{1} \mathrm{NS}=$ pasture during the day and cassava bagasse during the night, S46 $=N S+$ supplement (soybean hulls) from the $46^{\text {th }}$ day after the beginning of the breeding season until the birth and S100 = NS + supplement (soybean hulls) from the $100^{\text {th }}$ day after the beginning of the breeding season until the birth.

*DEV = Deviance to $D B 30, D B 60$.

\section{Conclusões}

Considerando o desempenho reprodutivo e produtivo, o uso de $0,5 \%$ do peso vivo de casca do grão de soja na alimentação das ovelhas não se justifica, quando estas recebem capim-Tanzânia mais resíduo de fécula de mandioca, nos moldes deste experimento.

A escolha da raça do reprodutor é facultativa neste modelo de produção, se considerados os pesos e mortalidade dos cordeiros até 60 dias de idade.

\section{Literatura Citada}

BARBIERE, M.E.; FIGUEIREDO, E.A.P.; SILVA, F.L.R.; et al. Avaliação de alguns parâmetros produtivos de ovinos Santa Inês, de pelagem preta. In: REUNIÃO ANUAL DA SOCIEDADE BRASILEIRA DE ZOOTECNIA, 28., 1991, João Pessoa. Anais... João Pessoa: Sociedade Brasileira de Zootecnia, 1991. p.594.

BUTLER-HOGG, B.W. The growth of Clun and Southdown sheep: body composition and the partitioning of total body fat. Animal Production, v.39, p.409-11, 1984.

CAMERON, N.D.; SMITH, C. Performance of lambs from three crossbred ewe types. Animal Production, v.39, p.81-87, 1984.

CHURCH, D.C. Alimentos y alimentacion del ganado. Montevideo: Hemisferio Sul, 1984.

CORRÊA, A.R. Forrageiras: Aptidão Climática do estado do Paraná. In: MONTEIRO A. L. G., MORAES, A., CORRÊA, E. A. S. et al. (Eds.) Forragicultura do Paraná. Londrina: CPAF, 1996. p.75-92.

DICKERSON, G. Experimental approaches in utilizing breed resources. Animal Breeding, n.37, p.191-202, 1969.

ESTRADA, L.H.C. Exigências nutricionais de ovinos para as condições brasileiras. In: CONGRESSO DA SOCIEDADE NORDESTINA DE PRODUÇÃO ANIMAL, 2., 2000, Teresina. Anais... Teresina: SNPA, 2000. v. 1. p.325-339.

FIGUEIRÓ, P.R.P. Manejo alimentar de ovinos. In: SIMPÓSIO PAULISTA DE OVINOCULTURA, 1., 1988. Botucatu. Anais...Campinas: Fundação Cargill, 1989. p.22-33.

GREENWOOD, P.L.; HUNT, A.S.; HERMANSON, J.W.; et al. Effects of birth weight ans post natal nutrition on neonatal sheep. II. Skeletal muscle growth and development. Journal Animal Science, n.78, p.50-61, 2000.

LIMA, D.; PIMENTA FILHO, E.C.; MALHEIROS FILHO, J.R. et al. Fatores ambientais que afetam o desenvolvimento ponderal de borregos Santa Inês. In: REUNIÃO ANUAL DA SOCIEDADE BRASILEIRA DE ZOOTECNIA, 28., 1991, João Pessoa. Anais... João Pessoa: Sociedade Brasileira de Zootecnia, 1991. p.486.

LIMA, F.A.M.; SILVA, F.L.R.; SANTOS, J.W. Desempenho produtivo de ovinos Santa Inês x Crioula, criados no Estado do Ceará. In: REUNIÃO ANUAL DA SOCIEDADE BRASILEIRA DE ZOOTECNIA, 24., 1987, Brasília. Anais... Brasília: Sociedade Brasileira de Zootecnia, 1987. p.322.

LOBATO, J.F.P.; BARCELLOS, J.O.J. Efeito na variação do peso vivo durante o acasalamento sobre o desempenho reprodutivo de novilhas de corte. In: REUNIÃO ANUAL DA SOCIEDADE BRASILEIRA DE ZOOTECNIA, 25, 1988, Viçosa, MG. Anais... Viçosa, MG: Sociedade Brasileira de Zootecnia, 1988. p.281.

LÔBO, R.N.B.; MARTINS FILHO, R.; FERNANDES, A.A.O. Efeitos de fatores genéticos e de ambiente sobre o peso ao nascimento de ovinos da raça Morada Nova no sertão do Ceará. Ciência Animal, v.2, n.1, p.95-104, 1992.

MACEDO, F.A.F. Desempenho e características de carcaças de cordeiros Corriedale mestiços Bergamácia $x$ Corriedale e Hampshire Down x Corriedale, terminados em pastagem e confinamento. Botucatu: Universidade Estadual Paulista, 1998. 72p. Tese (Doutorado em Zootecnia) - Universidade Estadual Paulista, 1998.

MACHADO, R.; SIMPLÍCIO, A.A.; BARBIERI, M.E. Acasalamento entre ovelhas deslanadas e reprodutores especializados para corte: Desempenho produtivo até a desmama. Revista Brasileira Zootecnia, v.28, n.4, p.706-712, 1999. 
McMANUS, C. Comparação das raças Santa Inês e Bergamácia no Distrito Federal: Características de adaptação em animais adultos. In: REUNIÃO ANUAL DA SOCIEDADE BRASILEIRA DE ZOOTECNIA, 32., 1995, Brasília. Anais... Brasília: Sociedade Brasileira de Zootecnia, 1995. p.136-137.

MUNIZ, E.N.; PIRES, C.C.; SILVA, J.H.S. et al. Efeito do número de cordeiros por parto e do sexo do cordeiro no crescimento ponderal. In: REUNIÃO ANUAL DA SOCIEDADE BRASILEIRA DE ZOOTECNIA, 34, 1997, Juiz de Fora. Anais... Juiz de Fora: Sociedade Brasileira de Zootecnia, 1997. p.266-268.

NATIONAL RESEARCH COUNCIL - NRC. Nutrients requirements of sheep. Washington, D.C.: National Academy Press, 1985. 99p.

OLIVEIRA, S.M.P.; MIRANDA, J.J.F.; PEREIRA, C.S. et al. Parâmetros genéticos e efeitos não genéticos dos pesos ao nascer, aos 112, aos 210 e aos 365 dias de idade de ovinos da raça Morada Nova variedade branca. In: REUNIÃO ANUAL DA SOCIEDADE BRASILEIRA DE ZOOTECNIA, 29., 1992, Lavras. Anais...Lavras: Sociedade Brasileira de Zootecnia, 1992. p.95.

PEETERS, R.; KOX, G.;ISTERDAEL, J.V. 1996. Environmental and maternal effects on early postnatal growth of lambs of different genotypes. Small Ruminant Research, n.19, p.45-53, 1996

PEREIRA, R.G.A.; MAGALHÃES, J.A.; COSTA, N.A. et al. Ovinos deslanados: Alternativa para a agricultura familiar. Resultados e discussão. In: REUNIÃO ANUAL DA SOCIEDADE BRASILEIRA DE ZOOTECNIA, 35., 1998, Botucatu. Anais... São Paulo: Gmosis, 1998, CD-ROM. Sistema de produção e economia. SIS-061.

RAMSEY, W.S.; HATFIELD, P.G.; WALLACE, J.D.; et al. 1994. Relationships among ewe milk production and ewe and lamb forage intake in Targhee ewes nursing single or twin lambs. Journal Animal Science, n.72, p.811-816, 1994.

RODRIGUES, F.E.; FERNANDES, L.C.O.; LÓPEZ, J.; et al. Efeito de três planos nutricionais na fertilidade e intervalo parto-cio em ovelhas Polwart (Ideal). In: REUNIÃO ANUAL DA SOCIEDADE BRASILEIRA DE ZOOTECNIA, 26., 1989, Porto Alegre. Anais... Porto Alegre: Sociedade Brasileira de Zootecnia, 1989. p.260.

SALOMÃO, J.A.F.; MIRANDA, R.M.; LOPES, H.O.S. Influência da suplementação com mistura protéica-energéticamineral no desempenho de ovelhas em final de gestação. In: REUNIÃO ANUAL DA SOCIEDADE BRASILEIRA DE ZOOTECNIA, 33., 1996, Fortaleza. Anais... Fortaleza: Sociedade Brasileira de Zootecnia, 1996. p.191-193.

SANTANA, A.F.S.; COSTA, G.B.; FONSECA, L.S. Correlações entre pesos e medidas corporais em ovinos jovens da raça Santa Inês. Revista Brasileira Saúde Produção Animal, n.1, p.74-77, 2001.

STATISTICAL ANALYSES SYSTEM - SAS. SAS Technical Report. Release 6.07. Cary: 1992, 229p.
SILVA, A.M.A.; ARRUDA, C.T. Avaliação da eficiência produtiva e reprodutiva de ovinos Santa Inês em pasto raleado no semi-árido paraibano. In: REUNIÃO ANUAL DA SOCIEDADE BRASILEIRA DE ZOOTECNIA, 25., 1988, Viçosa, MG. Anais... Viçosa, MG: Sociedade Brasileira de Zootecnia, 1988. p.306.

SILVA, D.J. Análise de alimentos: métodos químicos e biológicos. Viçosa, MG: Universidade Federal de Viçosa, 1990. 165p

SILVA, F.L.R.; FIGUEIREDO, E.A.P., BARBIERI, M.E. et al. Efeitos ambientais e de reprodutor sobre características de crescimento e de reprodução em ovinos Santa Inês, no Estado do Ceará. Revista Brasileira de Zootecnia, v.24,n.4,p.559-568, 1995.

SILVA. F.L.R.; ARAÚJO, A.M.; FIGUEIREDO, E.A. Características de crescimento e reprodução em ovinos Somalis no Nordeste brasileiro. Revista Brasileira de Zootecnia, v.27, n.6, p.1107-1114, 1998.

SILVEIRA, V.C.P.; LÓPEZ, J.; RODRIGUES, F.E. Influência da nutrição materna e do sexo na reserva energética do cordeiro ao nascer. Revista Brasileira de Zootecnia, v.21, n.2, p.242-249, 1992.

SOUZA JR., F.A.; SELAIVE-VILLARROEL, A.B.; MARTINS FILHO, R.; et al. Características de crescimento e de carcaça em três genótipos de cordeiros cruzas F1 mantidos em semiconfinamento, no Estado do Ceará. In: REUNIÃO ANUAL DA SOCIEDADE BRASILEIRA DE ZOOTECNIA, 37. 2000, Viçosa. Anais... São Paulo: Gmosis, 2000. CD-ROM. Sistema de produção e economia. SIS-1091.

SUSIN, I. Exigências nutricionais de ovinos e estratégias de alimentação. In: Nutrição de Ovinos. Jaboticabal: FUNEP, 1996. p.119-142.

VINAGRE, O.T.; SILVEIRA, J.B.; SIMPLÍCIO, J.B. et al. Parâmetros de avaliação do comportamento reprodutivo de ovinos Santa Inês, no Agreste Paraíbano. In: REUNIÃO ANUAL DE ZOOTECNIA, 29., 1992, Lavras. Anais... Lavras: Sociedade Brasileira de Zootecnia, 1992. p.231.

VINAGRE, O.T.; SILVEIRA, J.B.; VINAGRE, A.M.R. et al. Desempenho reprodutivo de ovelhas Santa Inês, no agreste paraibano. In: REUNIÃO ANUAL DA SOCIEDADE BRASILEIRA DE ZOOTECNIA, 26., 1989, Porto Alegre. Anais... Porto Alegre: Sociedade Brasileira de Zootecnia, 1989. p.259.

WOLF, B.T.; SMITH, C.; JALES, D.I. Growth and carcass composition in the crossbred progeny of six terminal sire breeds of sheep. Animal Production, v.31, p.307-313, 1980.

Recebido em: 14/10/02

Aceito em: 03/10/03 